\title{
Stability analysis for optimal control problems governed by semilinear evolution equation
}

Hongyong Deng ${ }^{1}$ and Wei Wei ${ }^{1,2^{*}}$

\author{
"Correspondence: \\ wwei@gzu.edu.cn \\ 'Department of Mathematics, \\ Guizhou University, Guiyang, \\ 550025, P.R. China \\ ${ }^{2}$ Department of Mathematics, \\ Guizhou Minzu University, Guiyang \\ 550025, P.R. China
}

\begin{abstract}
In this paper, the stability of solutions of optimal control for the distributed parameter system governed by a semilinear evolution equation with compact control set in the space $L^{1}(0, T ; E)$ is discussed. The stability results for optimal control problems with respect to the right-hand side functions are obtained by the theory of set-valued mapping and the definition of essential solutions for optimal control problems.
\end{abstract}

Keywords: optimal control; stability; semilinear evolution equation; $C_{0}$-semigroup; set-valued mapping

\section{Introduction}

The stability analysis of systems governed by differential equations in modern mathematics is very important for practical applications (see $[1,2])$, especially in numerical computation (see $[3,4])$.

In recent years, there has been growing interest in stability analysis of optimal control problems for the ODE system or PDE system (see [5, 6]). But most of the results are based on the existence and uniqueness of an optimal control for problems, there are only a small number of articles discussing a stability analysis for optimal control problems without the uniqueness in view of set-valued analysis. In recent years, $\mathrm{Yu}$ et al. discussed the stability of optimal controls with respect to the right-hand side function based on set-valued mapping (see [7]). But all results are established under the control admissible set $\mathcal{U}[0, T]$ assumed as the compact set of $C\left([0, T] ; R^{m}\right)$ in the optimal control problem for an ODE system. Moreover, we have done some work concerning the control admissible set $\mathcal{U}[0, T]$ assumed as the compact set of $L^{1}\left(0, T ; R^{m}\right)$ in order to include many cases of practical situations (see [8]). Therefore, it is natural for us to ask whether these results are still valid for infinite dimensional controlled systems.

This paper studies the existence and stability properties of solutions of optimal control problems governed by a semilinear evolution equation, and it is stated as follows.

Problem (P): Given $T>0, \hat{y}(\cdot) \in L^{2}(0, T ; X), y_{0} \in X$, and $y_{T} \in X$, find an optimal control $\bar{u} \in \mathcal{U}[0, T]$ such that

$$
J(\bar{u}) \leq J(u) \quad \text { for all } u \in \mathcal{U}[0, T],
$$

where

$$
J(u)=\left\|y(T)-y_{T}\right\|_{X}^{2}+\int_{0}^{T}\|y(t)-\hat{y}(t)\|_{X}^{2} d t
$$

\section{Springer}

( 2015 Deng and Wei; licensee Springer. This is an Open Access article distributed under the terms of the Creative Commons Attribution License (http://creativecommons.org/licenses/by/4.0), which permits unrestricted use, distribution, and reproduction in any medium, provided the original work is properly credited. 
subject to

$$
\left\{\begin{array}{l}
\dot{y}(t)=A y(t)+f(t, y(t), u(t)), \quad t \in[0, T] \\
y(0)=y_{0}
\end{array}\right.
$$

where $A$ is infinitesimal generator of $C_{0}$-semigroup on a Banach space $X$, the set of admissible controls in the space $L^{1}(0, T ; E)$ is defined by

$$
\mathcal{U}[0, T]=\{u \mid u(t) \text { is measurable w.r.t. } t, u(t) \in U \subset E\}
$$

where $E$ is another separable reflexive Banach space from which the controls $u$ take values.

The paper is organized as follows. In Section 2, we give some properties of $C_{0}$-semigroup and some results as regards compact sets. In Section 3, the existence of an optimal control is obtained. In Section 4, for the reader's convenience, the set-valued mapping theory is recalled, then we show stability results for the optimal control problem in the sense of the Baire category. In the last section, some examples demonstrate the applicability of our results.

\section{Preliminaries}

Throughout the paper, constants $1 \leq p<+\infty$ and $T>0$ are given. Let $X$ be a Banach space and $e^{A t}$ the $C_{0}$-semigroup generated by a linear operator $A$. In this section, we recall some related results about semigroups and compact sets from $[9,10]$ and $[11]$ which will be used in this paper.

Proposition 2.1 ([9]) There exist constants $\omega \geq 0$ and $M \geq 1$ such that

$$
\left\|e^{A t}\right\| \leq M e^{\omega t} \text { for } 0 \leq t<+\infty .
$$

Denote $L^{p}(0, T ; X)$ the space of measurable functions from $[0, T]$ into $X$ equipped with the norm

$$
\|y\|_{L^{p}(0, T ; X)}=\left(\int_{0}^{T}\|y(t)\|_{X}^{p} d t\right)^{\frac{1}{p}}
$$

and $C([0, T] ; X)$ the space of continuous functions from $[0, T]$ into $X$ equipped with the norm

$$
\|y\|_{C([0, T] ; X)}=\sup _{t \in[0, T]}\|y(t)\|_{X} .
$$

Lemma 2.2 ([11]) A set $V$ is called totally bounded if for every $\epsilon>0$, there exist some $\delta>0$, a metric space $W$, and a mapping $\Psi: V \rightarrow W$ such that $\Psi(V)$ is totally bounded, and $d(x, y)<\epsilon$ if $d(\Psi(x), \Psi(y))<\delta$ whenever $x, y \in V$.

Denote $\left(\tau_{h} u\right)(t)=u(t+h)$ for $h>0$, the compactness of $L^{p}(0, T ; X)$ is recalled as follows.

Lemma 2.3 ([12]) Suppose that $S$ is a subset of $L^{p}(0, T ; X)$. $S$ is relatively compact in $L^{p}(0, T ; X)$ if and only if: 
(1) The set $\left\{\int_{t_{1}}^{t_{2}} u(t) d t \mid u \in S\right\}$ is relatively compact in $X$, for any $0<t_{1}<t_{2}<T$.

(2) The limit $\left\|\tau_{h} u-u\right\|_{L^{p}(0, T-h ; X)} \rightarrow 0$ as $h \rightarrow 0$, uniformly for $u \in S$.

Now we consider the compactness of the admissible set (4).

Proposition 2.4 Suppose that $U \subseteq E$ is compact and $\left\|\tau_{h} u-u\right\|_{L^{1}(0, T-h ; E)} \rightarrow 0$ as $h \rightarrow 0$ uniformly for $u \in \mathcal{U}[0, T]$. Then $\mathcal{U}[0, T]$ is compact in $L^{1}(0, T ; E)$.

Proof It follows from the compactness of $U \subseteq E$ that there exists a constant $M>0$ such that

$$
\|\omega\|_{E} \leq M \quad \text { for all } \omega \in U
$$

hence for any $0<t_{1}<t_{2}<T$, we get

$$
\left\|\int_{t_{1}}^{t_{2}} u(t) d t\right\|_{E} \leq \int_{t_{1}}^{t_{2}}\|u(t)\|_{E} d t \leq \int_{0}^{T}\|u(t)\|_{E} d t \leq \int_{0}^{T} M d t \leq T M
$$

for all $u \in \mathcal{U}[0, T]$, this implies that $\int_{t_{1}}^{t_{2}}\|u(t)\|_{E} d t$ is bounded in $R^{1}$, so the set $\left\{\int_{t_{1}}^{t_{2}}\|u(t)\|_{E} d t \mid u \in \mathcal{U}[0, T]\right\}$ is relatively compact in $R^{1}$. Since $\int_{t_{1}}^{t_{2}} u(t) d t \in E$, by the HahnBanach theorem, there exists a bounded linear functional $P \in E^{*}$ such that

$$
\|P\|=1, \quad P(u)=\|u\|_{E} .
$$

By the continuity and linearity of $P$, we have

$$
P\left(\int_{t_{1}}^{t_{2}}\left(u_{1}(t)-u_{2}(t)\right) d t\right)=\int_{t_{1}}^{t_{2}} P\left(u_{1}(t)-u_{2}(t)\right) d t=\int_{t_{1}}^{t_{2}}\left\|u_{1}(t)-u_{2}(t)\right\|_{E} d t
$$

and

$$
\begin{aligned}
\left\|\int_{t_{1}}^{t_{2}}\left(u_{1}-u_{2}\right) d t\right\|_{E} & \leq \int_{t_{1}}^{t_{2}}\left\|u_{1}-u_{2}\right\|_{E} d t \\
& =\int_{t_{1}}^{t_{2}} P\left(u_{1}(t)-u_{2}(t)\right) d t \\
& =P\left(\int_{t_{1}}^{t_{2}}\left(u_{1}(t)-u_{2}(t)\right) d t\right),
\end{aligned}
$$

namely,

$$
\left\|\int_{t_{1}}^{t_{2}} u_{1}(t) d t-\int_{t_{1}}^{t_{2}} u_{2}(t) d t\right\|_{E} \leq\left|P\left(\int_{t_{1}}^{t_{2}} u_{1}(t) d t\right)-P\left(\int_{t_{1}}^{t_{2}} u_{2}(t) d t\right)\right| .
$$

Due to $\left\{\int_{t_{1}}^{t_{2}}\|u(t)\|_{E} d t \mid u \in \mathcal{U}[0, T]\right\}=\left\{P\left(\int_{t_{1}}^{t_{2}} u(t) d t\right) \mid u \in \mathcal{U}[0, T]\right\}$ is relatively compact in $R^{1}$ and inequality (6), by Lemma 2.2 , we see that $\left\{\int_{t_{1}}^{t_{2}} u(t) d t \mid u \in \mathcal{U}[0, T]\right\}$ is relatively compact in $E$, for any $0<t_{1}<t_{2}<T$.

To this end, by Lemma $2.3, \mathcal{U}[0, T]$ is relatively compact in $L^{1}(0, T ; E)$. We only need next to prove that $\mathcal{U}[0, T]$ is closed. 
If $\left\{u_{n}\right\} \subseteq \mathcal{U}[0, T]$ and $u_{n} \rightarrow u$ in $L^{1}(0, T ; E)$, namely,

$$
\int_{0}^{T}\left\|u_{n}(t)-u(t)\right\|_{E} d t \rightarrow 0
$$

then $\|u(t)\|_{E}$ is measurable in $[0, T]$ and there exists a subsequence of $\left\{u_{n}\right\}$, denoted by $\left\{u_{n_{k}}\right\}$, such that

$$
\left\|u_{n_{k}}(t)-u(t)\right\|_{E} \rightarrow 0 \quad \text { a.e. } t \in[0, T] \text { as } k \rightarrow+\infty
$$

i.e., $\left\|u_{n_{k}}(t)-u(t)\right\|_{E} \rightarrow 0$ for all $t \in A$, where $A$ is some subset of $[0, T]$ and $\mu([0, T] \backslash A)=0$.

Since $U$ is compact, for any fixed $t \in A$ and $u_{n_{k}}(t) \in U$, there exists $u(t) \in U$ such that

$$
u_{n_{k}}(t) \rightarrow u(t) \quad \text { in } E \text { as } k \rightarrow+\infty \text {. }
$$

Without loss of generality, we define

$$
\hat{u}(t)= \begin{cases}u(t), & t \in A, \\ u\left(t_{0}\right), & t \in[0, T] \backslash A,\end{cases}
$$

where $t_{0}$ can be chosen by any point of $A$, then $u(t)=\hat{u}(t)$ a.e. $t \in[0, T]$. It is clear that $\hat{u}(t) \in U$ for all $t \in[0, T] . u$ and $\hat{u}$ are equivalent in $L^{1}(0, T ; E)$. Namely,

$$
u_{n_{k}} \rightarrow \hat{u} \quad \text { in } L^{1}(0, T ; E) \text { as } k \rightarrow+\infty
$$

then $\mathcal{U}[0, T]$ is closed. From the above, we complete this proposition.

We give an example of the compact set in $L^{1}(0, T ; E)$ in the following.

Example 2.1 Let $u \in \mathcal{U}[0, T] \subset L^{1}\left(0, T ; W^{1, p}(\Omega)\right)$. For any fixed $x \in \Omega, u(t, x)$ is a piecewise continuous with respect to $t$ containing only a finite number of discontinuous points. $S(x)=\{u(t, x) \mid t \in[0, T]\}, S(x)$ is a bounded closed set in $W^{1, p}(\Omega)$, where $\Omega$ is a bounded open subset of $R^{n}$ and $\partial \Omega$ is $C^{1}$. Then $\mathcal{U}[0, T]$ is compact in $L^{1}\left(0, T ; L^{p}(\Omega)\right)$.

Proof From the compact embedding theorem of [13], that is, $W^{1, p}(\Omega)$ is compactly embedded in $L^{p}(\Omega)$, written $W^{1, p}(\Omega) \subset \subset L^{p}(\Omega)$, since $S(x)$ is a bounded closed set in $W^{1, p}(\Omega)$, then $S(x)$ is compact in $L^{p}(\Omega)$.

Suppose that $D=\left\{t_{1}, \ldots, t_{N}\right\} \subset[0, T]$ and $t_{0}=0<t_{1}<t_{2}<\cdots<t_{N}<T=t_{N+1}$. We define

$$
\tilde{u}(t, x)= \begin{cases}u(t, x), & t \in[0, T] \backslash\left\{t_{1}, \ldots, t_{N}\right\}, x \in \Omega, \\ u\left(t_{i}-0, x\right), & t=t_{i}, i=1,2,3, \ldots, N, x \in \Omega .\end{cases}
$$

Without loss of generality, we may assume $h>0$, then

$$
\begin{aligned}
& \left\|\tau_{h} u-u\right\|_{L^{1}\left(0, T-h ; L^{p}(\Omega)\right)} \\
& \quad=\int_{0}^{T-h}\|u(t+h)-u(t)\|_{L^{p}(\Omega)} d t
\end{aligned}
$$




$$
\begin{aligned}
& =\sum_{i=1}^{N} \int_{t_{i-1}}^{t_{i}}\|u(t+h)-u(t)\|_{L^{p}(\Omega)} d t+\int_{t_{N}}^{T-h}\|u(t+h)-u(t)\|_{L^{p}(\Omega)} d t \\
& =\sum_{i=1}^{N+1} \int_{t_{i-1}}^{t_{i}-h}\|\tilde{u}(t+h)-\tilde{u}(t)\|_{L^{p}(\Omega)} d t+\sum_{i=1}^{N} \int_{t_{i}-h}^{t_{i}}\|\tilde{u}(t+h)-\tilde{u}(t)\|_{L^{p}(\Omega)} d t \\
& \leq \sum_{i=1}^{N+1} \int_{t_{i-1}}^{t_{i}-h}\|\tilde{u}(t+h)-\tilde{u}(t)\|_{L^{p}(\Omega)} d t+2 M N h .
\end{aligned}
$$

It follows from the continuity of $u$ in $\left(t_{i-1}, t_{i}\right)(i=1,2,3, \ldots, N+1)$ that, for any fixed $h>0$, $\tilde{u}(\cdot)$ is uniform continuous in $\left[t_{i-1}, t_{i}-h\right](i=1,2,3, \ldots, N+1)$. That is, for any $t \in\left[t_{i-1}, t_{i}-h\right]$ and any $\epsilon>0$, there exists $\delta_{i}>0$ such that

$$
\|\tilde{u}(t+h)-\tilde{u}(t)\|_{L^{p}(\Omega)}<\frac{\epsilon}{2 T N}
$$

holds whenever $h<\delta_{i}$. Let $\delta=\min _{1 \leq i \leq N+1} \delta_{i}$, then when $h<\delta$, we have

$$
\|\tilde{u}(t+h)-\tilde{u}(t)\|_{L^{p}(\Omega)}<\frac{\epsilon}{2 T N} \quad \text { for all } t \in[0, T] \backslash\left\{t_{1}, \ldots, t_{N}\right\} .
$$

Hence

$$
\sum_{i=1}^{N+1} \int_{t_{i-1}}^{t_{i}-h}\|\tilde{u}(t+h)-\tilde{u}(t)\|_{L^{p}(\Omega)} d t \leq \frac{\epsilon}{2} .
$$

If we take $h<\min \left\{\delta, \frac{\epsilon}{4 M N}\right\}$, then

$$
\left\|\tau_{h} u-u\right\|_{L^{1}\left(0, T-h ; L^{p}(\Omega)\right)} \leq \frac{\epsilon}{2}+2 M N h \leq \frac{\epsilon}{2}+\frac{\epsilon}{2}=\epsilon .
$$

By Proposition 2.4, $\mathcal{U}[0, T]$ is compact in $L^{1}\left(0, T ; L^{p}(\Omega)\right)$.

\section{Existence of optimal control}

In order to study the optimal control problem (P), we assume that:

$\left(\mathrm{H}_{u}\right)$ The set $U \subseteq E$ is compact, $\mathcal{U}[0, T] \subset L^{1}(0, T ; E)$, and

$$
\left\|\tau_{h} u-u\right\|_{L^{1}(0, T-h ; E)} \rightarrow 0 \quad \text { as } h \rightarrow 0,
$$

uniformly for $u \in \mathcal{U}[0, T]$.

$\left(\mathrm{H}_{f}\right)$ The function $f:[0, T] \times X \times U \rightarrow X$ is continuous w.r.t. $t$ and $u$. There exist a function $L(t)>0$ and constant $C>0$ such that

$$
\begin{aligned}
& \|f(t, x, u)-f(t, y, u)\|_{X} \leq L(t)\|x-y\|_{X}, \\
& \|f(t, x, u)\|_{X} \leq C
\end{aligned}
$$

for all $x, y \in X, t \in[0, T], u \in U$.

$\left(\mathrm{H}_{A}\right)$ The operator $A: D(A) \subseteq X \rightarrow X$ generates a $C_{0}$-semigroup $e^{A t}$ on $X$. 
Definition 3.1 For given $u \in \mathcal{U}[0, T]$, a function $y:[0, T] \rightarrow X$ is called a mild solution of (3) if $y \in C([0, T] ; X)$ satisfies

$$
y(t)=e^{A t} y_{0}+\int_{0}^{t} e^{A(t-s)} f(s, y(s), u(s)) d s, \quad t \in[0, T] .
$$

This solution is denoted by $y(\cdot, u(\cdot))$.

From Proposition 5.3 of [10], we have the following theorem.

Theorem 3.1 Suppose assumptions $\left(\mathrm{H}_{f}\right),\left(\mathrm{H}_{A}\right)$, and $\left(\mathrm{H}_{u}\right)$ hold. Then, for any $u \in \mathcal{U}[0, T]$, the Cauchy problem (3) has a unique mild solution $y \in C([0, T] ; X)$.

Moreover, we also have the following result.

Theorem 3.2 Suppose assumptions $\left(\mathrm{H}_{f}\right),\left(\mathrm{H}_{A}\right)$, and $\left(\mathrm{H}_{u}\right)$ hold. $y \in C([0, T] ; X)$ is the mild solution of system (3), then the map $u(\cdot) \rightarrow y(\cdot, u(\cdot))$ is continuous from $L^{1}(0, T ; E)$ into $C([0, T] ; X)$.

Proof Suppose $y_{k}(t)$ and $y(t)$ are mild solutions of the system (3) with respect to $u_{k}$ and $u$, respectively. Then

$$
y_{k}(t)=e^{A t} y_{0}+\int_{0}^{t} e^{A(t-s)} f\left(s, y_{k}(s), u_{k}(s)\right) d s
$$

and

$$
y(t)=e^{A t} y_{0}+\int_{0}^{t} e^{A(t-s)} f(s, y(s), u(s)) d s .
$$

Since

$$
u_{k} \rightarrow u \quad \text { in } L^{1}(0, T ; E) \text { as } k \rightarrow+\infty
$$

by the Chebyshev inequality of Theorem 2.5 .3 in [14], we have

$$
\left\|u_{k}(t)-u(t)\right\|_{E} \rightarrow 0 \quad \text { for almost all } t \in[0, T] \text { as } k \rightarrow+\infty
$$

and the function $f$ is continuous w.r.t. $u$, it follows from Corollary 2.2.6 of [14] that

$$
\left\|f\left(t, y, u_{k}\right)-f(t, y, u)\right\|_{X} \rightarrow 0 \quad \text { for almost all } t \in[0, T] \text { as } k \rightarrow+\infty \text {, }
$$

and combining the Riesz Theorem 2.2.5(i) and Theorem 2.2.3 of [14], then for any subsequence $\left\{u_{k^{\prime}}\right\}$ we can extract a further subsequence $\left\{u_{k^{\prime \prime}}\right\}$ such that

$$
\left\|u_{k^{\prime \prime}}(t)-u(t)\right\|_{E} \rightarrow 0 \quad \text { a.e. } t \in[0, T]
$$

then

$$
\left\|f\left(t, y(t), u_{k^{\prime \prime}}(t)\right)-f(t, y(t), u(t))\right\|_{X} \rightarrow 0 \quad \text { a.e. } t \in[0, T] .
$$


Also from Proposition 2.1, there exist constants $\omega \geq 0$ and $M \geq 1$ such that

$$
\left\|e^{A t}\right\| \leq M e^{\omega t} \text { for } 0 \leq t \leq T
$$

Due to assumption $\left(\mathrm{H}_{f}\right)$ and the Lebesgue dominated convergence theorem, it follows that

$$
\lim _{k^{\prime \prime} \rightarrow \infty} \int_{0}^{T} M e^{\omega T}\left\|f\left(s, y(s), u_{k^{\prime \prime}}(s)\right)-f(s, y(s), u(s))\right\|_{X} d s=0 .
$$

Since the subsequence of $\left\{u_{k^{\prime}}\right\}$ is arbitrary, we can conclude that

$$
\lim _{k \rightarrow \infty} \int_{0}^{T} M e^{\omega T}\left\|f\left(s, y(s), u_{k}(s)\right)-f(s, y(s), u(s))\right\|_{X} d s=0,
$$

namely, for any $\epsilon>0$, there exists $N>0$ such that

$$
\int_{0}^{T} M e^{\omega T}\left\|f\left(s, y(s), u_{k}(s)\right)-f(s, y(s), u(s))\right\|_{X} d s \leq \epsilon
$$

holds whenever $k \geq N$. So

$$
\begin{aligned}
\left\|y_{k}(t)-y(t)\right\|_{X}= & \left\|\int_{0}^{t} e^{A(t-s)}\left[f\left(s, y_{k}(s), u_{k}(s)\right)-f(s, y(s), u(s))\right] d s\right\|_{X} \\
\leq & \int_{0}^{t} M e^{\omega T}\left\|\left[f\left(s, y_{k}(s), u_{k}(s)\right)-f\left(s, y(s), u_{k}(s)\right)\right]\right\|_{X} d s \\
& +\int_{0}^{T} M e^{\omega T}\left\|\left[f\left(s, y(s), u_{k}(s)\right)-f(s, y(s), u(s))\right]\right\|_{X} d s \\
\leq & M e^{\omega T} \int_{0}^{t} L(t)\left\|y_{k}(t)-y(t)\right\|_{X} d s+\epsilon,
\end{aligned}
$$

and using the Gronwall inequality, we obtain

$$
\left\|y_{k}(t)-y(t)\right\|_{X} \leq \epsilon e^{M e^{\omega T} \int_{0}^{t} L(s) d s} \leq \epsilon e^{M T e^{\omega T} \int_{0}^{T} L(s) d s}, \quad \forall t \in[0, T] .
$$

That is, $y\left(\cdot, u_{k}\right) \rightarrow y(\cdot, u) \in C([0, T] ; X)$.

From Proposition 2.4, we have the following lemma.

Lemma 3.3 If assumption $\left(\mathrm{H}_{u}\right)$ holds, $\mathcal{U}[0, T]$ is compact in $L^{1}(0, T ; E)$.

Now, we discuss the existence of an optimal control for problem (P).

Theorem 3.4 Suppose assumptions $\left(\mathrm{H}_{u}\right),\left(\mathrm{H}_{A}\right)$, and $\left(\mathrm{H}_{f}\right)$ hold, then problem $(\mathrm{P})$ admits at least one optimal control.

Proof Assume that there exists a minimizing sequence of $\left\{u_{k}\right\} \subseteq \mathcal{U}[0, T]$ such that

$$
\lim _{k \rightarrow \infty} J\left(u_{k}\right)=\inf _{u \in \mathcal{U}[0, T]} J(u)
$$


Since $\mathcal{U}[0, T]$ is compact, there exists a subsequence $\left\{u_{k^{\prime}}\right\}$ of $\left\{u_{k}\right\}$ and $\bar{u} \in \mathcal{U}[0, T]$ such that

$$
u_{k^{\prime}} \rightarrow \bar{u} \quad \text { in } L^{1}(0, T ; E) \text { as } k^{\prime} \rightarrow+\infty
$$

From Theorem 3.2 we have

$$
y_{u_{k^{\prime}}}(\cdot) \rightarrow y_{\bar{u}}(\cdot) \quad \text { in } C([0, T] ; X) \text { as } k^{\prime} \rightarrow+\infty \text {, }
$$

where $y_{u_{k^{\prime}}}(\cdot, \cdot)$ and $y_{\bar{u}}(\cdot, \cdot)$ are solutions of (3) with respect to $u_{k^{\prime}}$ and $\bar{u}$, respectively. Hence

$$
y_{u_{k^{\prime}}}(T) \rightarrow y_{\bar{u}}(T) \quad \text { in } X \text { as } k^{\prime} \rightarrow+\infty
$$

and

$$
\left\|y_{u_{k^{\prime}}}(T)-y_{T}\right\|_{X} \rightarrow\left\|y_{\bar{u}}(T)-y_{T}\right\|_{X} \quad \text { in } R^{1} \text { as } k^{\prime} \rightarrow+\infty,
$$

then

$$
\left\|y_{u_{k^{\prime}}}(T)-y_{T}\right\|_{X}^{2} \rightarrow\left\|y_{\bar{u}}(T)-y_{T}\right\|_{X}^{2} \quad \text { in } R^{1} \text { as } k^{\prime} \rightarrow+\infty .
$$

Thanks to (15), there exists a subsequence of $\left\{u_{k^{\prime}}\right\}$, denoted by $\left\{u_{k^{\prime \prime}}\right\}$, such that

$$
\left\|u_{k^{\prime \prime}}(t)-\bar{u}(t)\right\|_{E} \rightarrow 0 \quad \text { for almost all } t \in[0, T] .
$$

It follows from (16) and (17) that

$$
\left\|y_{u_{k^{\prime \prime}}}(t)-\hat{y}(t)\right\|_{X} \rightarrow\left\|y_{\bar{u}}(t)-\hat{y}(t)\right\|_{X} \quad \text { a.e. } t \in[0, T] .
$$

Since $u_{k^{\prime \prime}}(t) \in U$ (compact set in $E$ ) and $y_{k^{\prime \prime}}(\cdot) \in C([0, T] ; X)$, the image of the compact set under $y$ is compact (see Theorem 2.5-6 of [15]). For all $k^{\prime \prime}$, there exists a positive constant $M$, such that

$$
\left\|y_{u_{k^{\prime \prime}}}(t)-\hat{y}(t)\right\|_{X} \leq M
$$

From the Lebesgue dominated convergence theorem (see Theorem 2.8.1 of [14]), we have

$$
\lim _{k^{\prime \prime} \rightarrow+\infty} \int_{0}^{T}\left\|y_{u_{k^{\prime \prime}}}(t)-\hat{y}(t)\right\|_{X}^{2} d t=\int_{0}^{T}\left\|y_{\bar{u}}(t)-\hat{y}(t)\right\|_{X}^{2} d t .
$$

From all the above, we have

$$
\begin{aligned}
J(\bar{u}) & =\left\|y_{\bar{u}}(T)-y_{T}\right\|_{X}^{2}+\int_{0}^{T}\left\|y_{\bar{u}}(t)-\hat{y}(t)\right\|_{X}^{2} d t \\
& =\lim _{k^{\prime \prime} \rightarrow+\infty}\left\|y_{u_{k^{\prime \prime}}}(T)-y_{T}\right\|_{X}^{2}+\lim _{k^{\prime \prime} \rightarrow+\infty} \int_{0}^{T}\left\|y_{u_{k^{\prime \prime}}}(t)-\hat{y}(t)\right\|_{X}^{2} d t \\
& =\lim _{k^{\prime \prime} \rightarrow+\infty} J\left(u_{k^{\prime \prime}}\right)=\inf _{u \in \mathcal{U}[0, T]} J(u) .
\end{aligned}
$$

That is, $\bar{u} \in \mathcal{U}[0, T]$ is an optimal control. This completes the proof of the theorem. 


\section{Stability analysis of optimal control problems}

In this section, we will use the set-valued mapping theory to study the stability of the optimal control. At first, we recall some definitions and a lemma on set-valued mappings for convenience of the reader (see $[7,8,16])$. Let $W$ and $Z$ be metric spaces.

Definition 4.1 A set-valued mapping $F: W \rightarrow 2^{Z}$ is called upper (respectively, lower) semicontinuous at $x \in W$ if and only if for each open set $G$ in $Z$ with $G \supset F(x)$ (respectively, $G \cap F(x) \neq \emptyset$ ), there exists $\delta>0$ such that $G \supset F\left(x^{\prime}\right)$ (respectively, $\left.G \cap F\left(x^{\prime}\right) \neq \emptyset\right)$ for any $x^{\prime} \in W$ with $\rho\left(x, x^{\prime}\right)<\delta$. It is said to be upper (respectively, lower) semicontinuous in $W$ if and only if it is upper (respectively, lower) semicontinuous at any point of $W$.

A set-valued mapping $F: W \rightarrow 2^{Z}$ is continuous at $x$ if it is both upper semicontinuous and lower semicontinuous at $x$, and that it is continuous if and only if it is continuous at every point of $W$.

Definition 4.2 A set-valued mapping $F: W \rightarrow 2^{Z}$ is called compact upper semicontinuous (called USCO) if $F(x)$ is nonempty compact, for each $x \in W$, and $F$ is upper semicontinuous.

Definition 4.3 A set-valued mapping $F: W \rightarrow 2^{Z}$ is called closed if $\operatorname{Graph}(F)$ is closed, where $\operatorname{Graph}(F)=\{(x, z) \in W \times Z \mid z \in F(x)\}$ is the graph of $F$.

Lemma 4.1 If the set-valued mapping $F: W \rightarrow 2^{Z}$ is closed and $Z$ is compact, then $F$ is an USCO mapping.

Definition 4.4 A subset $Q \subset W$ is called a residual set if it contains a countable intersection of open dense subsets of $W$.

If $W$ is a complete metric space, any residual subset of $W$ must be dense in $W$.

Lemma 4.2 Let $W$ be a complete metric space, and $F: W \rightarrow 2^{Z}$ be an USCO mapping, then there exists a dense residual subset $Q$ of $W$ such that $F$ is lower semicontinuous at each $x \in Q$.

Now, we consider the stability of optimal controls. Denote

$$
Y=\left\{f \mid f \text { satisfies conditions of }\left(\mathrm{H}_{f}\right)\right\} \text {. }
$$

For every $f_{1}, f_{2} \in Y$, we define

$$
\rho\left(f_{1}, f_{2}\right)=\sup _{(t, y, u) \in[0, T] \times X \times U}\left\|f_{1}(t, y, u)-f_{2}(t, y, u)\right\|_{X}
$$

Then the space $(Y, \rho)$ is a complete metric space [7].

Let

$$
S(f)=\{\bar{u} \mid \bar{u} \text { is the optimal control of problem (P) associated with } f \in Y\} .
$$

Then the correspondence $f \rightarrow S(f)$ is a set-valued mapping $S: Y \rightarrow 2^{\mathcal{U}[0, T]}$.

From Theorem 3.4, we have the following theorem. 
Theorem 4.3 If assumptions $\left(\mathrm{H}_{u}\right),\left(\mathrm{H}_{A}\right)$, and $\left(\mathrm{H}_{f}\right)$ hold, $S(f) \neq \emptyset$ for each $f \in Y$.

The following proposition is important in studying the stability of optimal controls.

Proposition 4.4 Let $\left\{f_{k}\right\}$ be any sequence of $Y$ such that $f_{k} \rightarrow f$ in $Y$ and $\left\{u_{k}\right\}$ any sequence of $\mathcal{U}[0, T]$ such that $u_{k} \rightarrow u$ in $L^{1}(0, T ; E)$, then $y_{f_{k}}\left(\cdot, u_{k}(\cdot)\right) \rightarrow y_{f}(\cdot, u(\cdot))$ in $C([0, T] ; X)$ as $k \rightarrow+\infty$.

Proof By Definition 3.1 of a mild solution of Cauchy problem (3) we have

$$
y(t) \triangleq y_{f}(t, u(t))=e^{A t} y_{0}+\int_{0}^{t} e^{A(t-s)} f(s, y(s), u(s)) d s
$$

and

$$
y_{k}(t) \triangleq y_{f_{k}}\left(t, u_{k}(t)\right)=e^{A t} y_{0}+\int_{0}^{t} e^{A(t-s)} f_{k}\left(s, y_{k}(s), u_{k}(s)\right) d s
$$

for every $t \in[0, T]$.

It follows from $f_{k} \rightarrow f$ in $Y$ that for any $\epsilon>0$ there exists a constant $N_{1}>0$ such that

$$
\rho\left(f_{k}, f\right) \leq \frac{\epsilon}{2 M T e^{\omega T}}
$$

whenever $k \geq N_{1}$. The inequality (8) implies that

$$
\begin{aligned}
& \int_{0}^{T}\left\|e^{A(T-s)}\left(f_{k}\left(s, y_{k}(s), u_{k}(s)\right)-f\left(s, y_{k}(s), u_{k}(s)\right)\right)\right\|_{X} d s \\
& \quad \leq \int_{0}^{T} M e^{\omega T}\left\|f_{k}\left(s, y_{k}(s), u_{k}(s)\right)-f\left(s, y_{k}(s), u_{k}(s)\right)\right\|_{X} d s \\
& \quad \leq \int_{0}^{T} M e^{\omega T} \frac{\epsilon}{2 M T e^{\omega T}} d s=\frac{\epsilon}{2} .
\end{aligned}
$$

The $u_{k} \rightarrow u$ in $L^{1}(0, T ; E)$ and (10) imply that there exists a constant $N_{2}>0$ such that

$$
\int_{0}^{T} M e^{\omega T}\left\|f\left(s, y(s), u_{k}(s)\right)-f(s, y(s), u(s))\right\|_{X} d s \leq \frac{\epsilon}{2}
$$

whenever $k \geq N_{2}$.

Let $N=\max \left\{N_{1}, N_{2}\right\}$, then

$$
\begin{aligned}
\left\|y_{k}(t)-y(t)\right\|_{X}= & \left\|\int_{0}^{t} e^{A(t-s)}\left[f_{k}\left(s, y_{k}(s), u_{k}(s)\right)-f(s, y(s), u(s))\right] d s\right\|_{X} \\
\leq & \int_{0}^{T} M e^{\omega T}\left\|f_{k}\left(s, y_{k}(s), u_{k}(s)\right)-f\left(s, y_{k}(s), u_{k}(s)\right)\right\|_{X} d s \\
& +\int_{0}^{t} M e^{\omega T}\left\|f\left(s, y_{k}(s), u_{k}(s)\right)-f\left(s, y(s), u_{k}(s)\right)\right\|_{X} d s \\
& +\int_{0}^{T} M e^{\omega T}\left\|f\left(s, y(s), u_{k}(s)\right)-f(s, y(s), u(s))\right\|_{X} d s \\
\leq & M e^{\omega T} \int_{0}^{t} L(t)\left\|y_{k}(t)-y(t)\right\|_{X} d s+\epsilon
\end{aligned}
$$


holds for any $k \geq N$, and it follows from the Gronwall inequality that

$$
\left\|y_{k}(t)-y(t)\right\|_{X} \leq \epsilon e^{M e^{\omega T} \int_{0}^{t} L(s) d s} \leq \epsilon e^{M T e^{\omega T} \int_{0}^{T} L(s) d s}, \quad \forall t \in[0, T] .
$$

This implies $y_{k} \rightarrow y$ in $C([0, T] ; X)$, which completes the proof of the theorem.

We denote

$$
J_{f_{k}}\left(u_{k}\right)=\left\|y_{k}(T)-y_{T}\right\|_{X}^{2}+\int_{0}^{T}\left\|y_{k}(t)-\hat{y}(t)\right\|_{X}^{2} d t .
$$

One can easily obtain the following proposition from Proposition 4.4.

Proposition 4.5 Let $\left\{f_{k}\right\}$ be any sequence of $Y$ such that $f_{k} \rightarrow f$ in $Y$ and $\left\{u_{k}\right\}$ any sequence of $\mathcal{U}[0, T]$ such that $u_{k} \rightarrow u$ in $L^{1}(0, T ; E)$. Then $J_{f_{k}}\left(u_{k}\right) \rightarrow J_{f}(u)$ as $k \rightarrow+\infty$.

Theorem 4.6 Suppose that $\left(\mathrm{H}_{u}\right),\left(\mathrm{H}_{A}\right)$, and $\left(\mathrm{H}_{f}\right)$ hold. Then $S: Y \rightarrow 2^{\mathcal{U}[0, T]}$ is an USCO mapping.

Proof By the compactness of $\mathcal{U}[0, T]$ and Lemma 4.1, we only need to show that

$$
\operatorname{Graph}(S)=\{(f, u) \in Y \times \mathcal{U}[0, T] \mid u \in S(f)\}
$$

is closed. Let $\left\{f_{k}\right\} \subset Y$ with $f_{k} \rightarrow f$ in $Y$ and $\left\{\bar{u}_{k}\right\} \subset S\left(f_{k}\right)$ with $\bar{u}_{k} \rightarrow \bar{u}$ in $L^{1}(0, T ; E)$. Now we need to show that $\bar{u} \in S(f)$.

Due to $\bar{u}_{k} \in S\left(f_{k}\right)$, we have

$$
J_{f_{k}}\left(\bar{u}_{k}\right) \leq J_{f_{k}}(u) \quad \text { for all } u \in \mathcal{U}[0, T]
$$

Proposition 4.5 yields

$$
J_{f_{k}}\left(\bar{u}_{k}\right) \rightarrow J_{f}(\bar{u})
$$

and

$$
J_{f_{k}}(u) \rightarrow J_{f}(u) \quad \text { for all } u \in \mathcal{U}[0, T]
$$

Combining (25), (26), and (24), we have

$$
J_{f}(\bar{u}) \leq J_{f}(u) \quad \text { for all } u \in \mathcal{U}[0, T]
$$

namely, $\bar{u} \in S(f)$. The proof is completed.

We introduce the following definition for considering the stability of solutions of an optimal control problem.

Definition $4.5 u \in S(f)$ is called an essential solution iff for any $\epsilon>0$ there exists $\delta>0$ such that for any $f^{\prime} \in Y$ with $\rho\left(f^{\prime}, f\right)<\delta$, there is $u^{\prime} \in S\left(f^{\prime}\right)$ with $\left\|u-u^{\prime}\right\|_{L^{1}(0, T ; E)}<\epsilon$. The 
optimal control problem (P) associated with $f$ is called essential iff its solutions are all essential.

From [7], we can obtain the following theorem.

Theorem 4.7 The optimal control problem $(\mathrm{P})$ associated with $f$ is essential if and only if $S: Y \rightarrow 2^{\mathcal{U}[0, T]}$ is lower semicontinuous at $f \in Y$.

Now we need to consider that $S$ is lower semicontinuous in $Y$. From Lemma 4.2 and Theorem 4.6, we have the following lemma.

Lemma 4.8 There exists a dense residual subset $Q \subset Y$ such that $S$ is lower semicontinuous at each $f \in Q$, namely, $S$ is continuous at each $f \in Q$.

Since $S$ is continuous at each $f \in Q$ and $Y$ is a complete metric space, $Q$ is a second category [17]. Lemma 4.8 and Theorem 4.7 yield the generic stability in the sense of the Baire category.

Theorem 4.9 There exists a dense residual subset $Q$ of $Y$ such that for any $f \in Q, S(f)$ is stable in the sense of Hausdorff metric and $J_{f}$ is robust with respect to $f \in Q$. So every optimal control problem associated $f \in Y$ can be closely approximated arbitrarily by an essential optimal control problem.

We need to note that when the solution $S(f)$ is a singleton, the result also holds. By Theorem 4.9 we can also see that any $f \in Y$ can be closely approximated by an essential optimal control problem.

Remark 4.1 In this paper, we only discuss the stability results as regards the optimal control problem with quadratic cost functional. In fact, the results also hold for the optimal control problem with general cost functional such as Bolza problems under some assumptions.

\section{Example}

Our main result can be applied to the controlled systems of heat equations and wave equations with the $C_{0}$-semigroup. We will state optimal control problems with parabolic controlled systems and hyperbolic controlled systems, respectively, in the following.

Problem (P1): Given $T>0, \hat{y}(t, x)$, and $y_{T}(x)$, find an optimal control $\bar{u} \in \mathcal{U}[0, T]$ such that

$$
J(\bar{u}) \leq J(u) \quad \text { for all } u \in \mathcal{U}[0, T]
$$

where

$$
J(u)=\int_{\Omega}\left|y(T, x)-y_{T}(x)\right|^{2} d x+\int_{0}^{T} \int_{\Omega}|y(t, x)-\hat{y}(t, x)|^{2} d x d t
$$

subject to

$$
\left\{\begin{array}{l}
\frac{\partial y}{\partial t}(t, x)=\Delta y(t, x)+f(t, y(t, x), u(t, x)), \quad t \in[0, T], x \in \Omega, \\
y(0, x)=y_{0}(x), \quad x \in \Omega \\
\left.y\right|_{\partial \Omega}=0
\end{array}\right.
$$


where $\Omega \subset R^{n}$ is a bounded domain with a smooth boundary $\partial \Omega$, let $X=L^{2}(\Omega)$ and $A=$ $\Delta=\frac{\partial^{2}}{\partial x_{1}^{2}}+\cdots+\frac{\partial^{2}}{\partial x_{n}^{2}}$ with $D(A)=W^{2,2}(\Omega) \cap W_{0}^{1,2}(\Omega)$. Then $A$ is infinitesimal generator of a $C_{0}$-semigroup $e^{A t}$ on the infinite dimensional Banach space $X$. Suppose that $y_{0} \in X$ and the set of admissible controls in the space $L^{1}(0, T ; E)$ is defined by $(4)$ where $E=L^{2}(\Omega)$. With the assumptions $\left(\mathrm{H}_{u}\right)$ and $\left(\mathrm{H}_{f}\right)$ holding, the stability results are valid.

Problem (P2): Given $T>0, \hat{y}(t, x)$, and $y_{T}(x)$, find an optimal control $\bar{u} \in \mathcal{U}[0, T]$ such that

$$
J(\bar{u}) \leq J(u) \quad \text { for all } u \in \mathcal{U}[0, T]
$$

where

$$
J(u)=\int_{\Omega}\left|y(T, x)-y_{T}(x)\right|^{2} d x+\int_{0}^{T} \int_{\Omega}|y(t, x)-\hat{y}(t, x)|^{2} d x d t
$$

subject to

$$
\left\{\begin{array}{l}
\frac{\partial^{2} y}{\partial t^{2}}(t, x)=\Delta y(t, x)+f(t, y(t, x), u(t, x)), \quad t \in[0, T], x \in \Omega \\
y(0, x)=y_{0}(x),\left.\quad \frac{\partial y}{\partial t}\right|_{t=0}=y_{1}(x), \quad x \in \Omega \\
\left.y\right|_{\partial \Omega}=0
\end{array}\right.
$$

where $\Omega \subset R^{n}$ is a bounded domain with a smooth boundary $\partial \Omega$ and $X=W^{1,2}(\Omega) \times L^{2}(\Omega)$. Let $\left(y_{0}, y_{1}\right) \in X, \Delta=\frac{\partial^{2}}{\partial x_{1}^{2}}+\cdots+\frac{\partial^{2}}{\partial x_{n}^{2}}, A=\left(\begin{array}{cc}0 & I \\ \Delta & 0\end{array}\right)$ with $D(A)=\left(W^{2,2}(\Omega) \cap W_{0}^{1,2}(\Omega)\right) \times W^{1,2}(\Omega)$. Then $A$ is the infinitesimal generator of a $C_{0}$-semigroup $e^{A t}$ on the infinite dimensional Banach space $X$, the set of admissible controls in the space $L^{1}(0, T ; E)$ is defined by $(4)$ where $E=L^{2}(\Omega)$. With the assumptions $\left(\mathrm{H}_{u}\right)$ and $\left(\mathrm{H}_{f}\right)$ holding, the stability results are also valid.

The following example is provided to show that not all optimal control problems are essential.

Example 5.1 Let $H=[0,1], \Omega=[0, \pi] \subset R^{1}$. Consider the optimal problem

$$
\min _{u \in \mathcal{U}[0,1]} J(u)=\int_{0}^{\pi}(v(1, x))^{2} d x
$$

subject to the distributed parameter system

$$
\left\{\begin{array}{l}
\frac{\partial}{\partial t} v(t, x)=\frac{\partial^{2}}{\partial x^{2}} v(t, x)+f(t, v(t, x), u(t, x)), \quad t \in H, x \in \Omega \\
v(t, 0)=v(t, \pi)=0, \quad t \in H \\
v(0, x)=v_{0}, \quad x \in \Omega
\end{array}\right.
$$

where $u \in \mathcal{U}[0,1], X=L^{2}(\Omega), v_{0} \in X, A=\frac{\partial^{2}}{\partial x^{2}}$, and $D(A)=W^{2,2}(\Omega) \cap W_{0}^{1,2}(\Omega)$. Then the $A$ generate a $C_{0}$-semigroup $e^{A t}$. The mild solution can be given by

$$
v(t, x)=e^{A t} v_{0}+\int_{0}^{t} e^{A(t-s)} f(s, v(s, x), u(s, x)) d s
$$


Let

$$
\mathcal{U}[0,1]=\left(\bigcup_{k=1}^{N}\left\{u_{k}(t, x) \in\left\{\sin x,-e^{-\frac{1}{2 k}} \sin x\right\}\right\}\right) \cup\left(\bigcup_{m=1}^{+\infty}\left\{u^{m}(t, x) \equiv-\frac{1}{m} \sin x\right\}\right),
$$

here we set

$$
u_{k}(t, x)= \begin{cases}\sin x & \text { if } t \in\left[\frac{2 l}{2 k}, \frac{2 l+1}{2 k}\right), l=0,1, \ldots, k-1, \\ -e^{-\frac{1}{2 k}} \sin x & \text { otherwise. }\end{cases}
$$

Similarly to Example $2.1, \mathcal{U}[0,1]$ is compact.

In order to simplify the calculation, let $v_{0}=0$.

(1) Let $f(t, v(t, x), u(t, x))=u(t, x) \in \mathcal{U}[0,1]$, we see that the $A$ generate a $C_{0}$-semigroup $T(t)=e^{-t}$; then the mild solution is

$$
v(t, x)=\int_{0}^{t} e^{-(t-s)} u(s, x) d s,
$$

we have $v(1, x)=0$, for any $u_{k}, k=1,2, \ldots, N$, hence $J\left(u_{k}\right)=0, k=1,2, \ldots, N$. So

$$
S(f)=\arg \min _{u \in \mathcal{U}[0,1]} J_{f}(u)=\left\{u_{k}, k=1,2, \ldots, N\right\}
$$

(2) Let $f_{m}(t, v(t, x), u(t, x))=u(t, x)+\frac{1}{m} \sin x, t \in H, x \in \Omega$, for every $m=1,2, \ldots$. Then $v(1, x)=0$, and $J\left(u_{m}\right)=0, m=1,2, \ldots$, we get

$$
S\left(f_{m}\right)=\arg \min _{u \in \mathcal{U}[0,1]} J_{f_{m}}(u)=\left\{u^{m}\right\}
$$

We see $\rho\left(f, f_{m}\right)=\frac{\sqrt{2 \pi}}{2 m} \rightarrow 0$ as $m \rightarrow+\infty$. For any $u_{k} \in S(f)$, we have

$$
\begin{aligned}
& \int_{0}^{1}\left\|u_{k}(t, x)-u^{m}(t, x)\right\|_{L^{2}(\Omega)} d t \\
& =\frac{\sqrt{2 \pi}}{2}\left[\int_{H_{1}}\left(1-\frac{1}{m}\right) d t+\int_{H_{2}}\left(e^{-\frac{1}{2 k}}+\frac{1}{m}\right) d t\right] \\
& =\frac{\sqrt{2 \pi}}{2}\left(1+e^{-\frac{1}{2 k}}\right),
\end{aligned}
$$

where $H_{1}$ is the union of intervals in which $u_{k}$ takes the value $\sin x$ and $H_{2}$ is the union of intervals in which $u_{k}$ takes the value $-e^{-\frac{1}{2 k}} \sin x$, which shows that $u_{k}$ is not essential. If let $u^{0}(t, x) \equiv 0$, we have

$$
\int_{0}^{1}\left\|u^{0}(t, x)-u^{m}(t, x)\right\|_{L^{2}(\Omega)} d t=\frac{\sqrt{2 \pi}}{2 m} \rightarrow 0 \quad \text { as } m \rightarrow \infty .
$$

Then all solutions in $S(f)$ except $u^{0}(t, x) \equiv 0$ are not essential. 
Authors' contributions

All authors contributed equally to the writing of this paper. All authors read and approved the final manuscript.

\section{Acknowledgements}

This work was supported by the National Natural Science Foundation of China (Grant No. 11261011).

Received: 15 October 2014 Accepted: 16 March 2015 Published online: 28 March 2015

\section{References}

1. Dontchev, AL, Hager, WW: Lipschitzian stability for state constrained nonlinear optimal control. SIAM J. Control Optim. 36, 698-718 (1998)

2. Hermant, A: Stability analysis of optimal control problems with a second-order constraint. SIAM J. Control Optim. 20, 104-129 (2009)

3. Teo, KL, Goh, CJ, Wong, KH: A Unified Computational Approach to Optimal Control Problems. Wiley, New York (1991)

4. Lin, Q, Loxton, R, Teo, KL: The control parameterization method for nonlinear optimal control: a survey. J. Ind. Manag. Optim. 10, 275-309 (2014)

5. Casas, E, Kunisch, K: Optimal control of semilinear elliptic equations in measure spaces. SIAM J. Control Optim. 52 339-364 (2014)

6. Corona, D, Giua, A, Seatzu, C: Stabilization of switched systems via optimal control. Nonlinear Anal. Hybrid Syst. 11, 1-10 (2014)

7. Yu, J, Liu, ZX, Peng, DT: Existence and stability analysis of optimal control. Optim. Control Appl. Methods 35, 721-729 (2014)

8. Deng, HY, Wei, W: Existence and stability analysis for nonlinear optimal control problems with 1-mean equicontinuous controls. J. Ind. Manag. Optim. 11, 1409-1422 (2015). doi:10.3934/jimo.2015.11.1409

9. Pazy, A: Semigroups of Linear Operators and Applications to Partial Differential Equations. Springer, New York (1983)

10. Li, XJ, Yong, JM: Optimal Control Theory for Infinite Dimensional Systems. Birkhäuser, Boston (1995)

11. Hanche-Olsen, H, Holden, H: The Kolmogorov-Riesz compactness theorem. Expo. Math. 28, 385-394 (2010)

12. Simon, J: Compact sets in the space $L^{P}(0, T ; B)$. Ann. Mat. Pura Appl. 146, 65-96 (1986)

13. Evans, LC: Partial Differential Equations. Am. Math. Soc., Providence (1998)

14. Bogachev, VI: Measure Theory, vol. I. Springer, Berlin (2007)

15. Kreyszig, E: Introductory Functional Analysis with Applications. Wiley, New York (1978)

16. Aubin, JP, Frankowska, H: Set-Valued Analysis. Birkhäuser, Boston (1990)

17. Rudin, W: Functional Analysis, 2nd edn. McGraw-Hill, New York (1991)

\section{Submit your manuscript to a SpringerOpen ${ }^{\circ}$ journal and benefit from:}

- Convenient online submission

Rigorous peer review

- Immediate publication on acceptance

- Open access: articles freely available online

- High visibility within the field

- Retaining the copyright to your article 